Published in: Stochastic Analysis and Applications 25 (2007), 6: 1273-1296

\title{
Continuous local time of a purely atomic immigration superprocess with dependent spatial motion
}

\author{
Zenghu $\mathbf{L i}^{1}$ and Jie Xiong ${ }^{2,3}$ \\ 1 School of Mathematical Sciences, Beijing Normal University, \\ Beijing 100875, P.R. China. E-mail: lizh@bnu.edu.cn \\ 2 Department of Mathematics, University of Tennessee, Knoxville, \\ TN 37996-1300, U.S.A. E-mail: jxiong@math.utk.edu \\ 3 Department of Mathematics, Hebei Normal University, \\ Shijiazhuang 050016, P.R. China
}

\begin{abstract}
A purely atomic immigration superprocess with dependent spatial motion in the space of tempered measures is constructed as the unique strong solution of a stochastic integral equation driven by Poisson processes based on the excursion law of a Feller branching diffusion, which generalizes the work of Dawson and Li [3]. As an application of the stochastic equation, it is proved that the superprocess possesses a local time which is Hölder continuous of order $\alpha$ for every $\alpha<1 / 2$. We establish two scaling limit theorems for the immigration superprocess, from which we derive scaling limits for the corresponding local time.
\end{abstract}

Key words: local time, superprocess, dependent spatial motion, immigration, excursion, Poisson random measure; scaling limit theorem.

Mathematics Subject Classification (2000): 60J80; 60G57;60H20

Running head: Continuous local time of immigration superprocess. 


\section{Introduction}

Let $p \geq 0$ and let $\phi_{p}(x)=\left(1+x^{2}\right)^{-p / 2}$ for $x \in \mathbb{R}$. We denote by $C_{p}(\mathbb{R})$ the set of continuous functions $\phi$ on $\mathbb{R}$ satisfying $|\phi| \leq$ const $\cdot \phi_{p}$ and denote by $C_{p}^{2}(\mathbb{R})$ the subset of $C_{p}(\mathbb{R})$ consisting of twice continuously differentiable functions $\phi$ with $\left|\phi^{\prime}\right|+\left|\phi^{\prime \prime}\right| \leq$ const $\cdot \phi_{p}$. Let $M_{p}(\mathbb{R})$ denote the space of tempered Borel measures $\mu$ on $\mathbb{R}$ such that

$$
\langle\phi, \mu\rangle:=\int_{\mathbb{R}} \phi(x) \mu(d x)<\infty
$$

for every $\phi \in C_{p}(\mathbb{R})$. Let $M_{p}^{a}(\mathbb{R})$ be the subset of $M_{p}(\mathbb{R})$ consisting of purely atomic measures. In the case $p=0$, we simply write $C(\mathbb{R})$ and $M(\mathbb{R})$ instead of $C_{0}(\mathbb{R})$ and $M_{0}(\mathbb{R})$, respectively. Let " $\|\cdot\|$ " denote the supremum norm. Suppose that $h$ is a continuously differentiable function on $\mathbb{R}$ such that both $h$ and $h^{\prime}$ are square-integrable. Then the function

$$
\rho(x)=\int_{\mathbb{R}} h(y-x) h(y) d y, \quad x \in \mathbb{R}
$$

is twice continuously differentiable with bounded derivatives $\rho^{\prime}$ and $\rho^{\prime \prime}$. We fix a constant $\sigma>0$ and a measure $m \in M_{p}(\mathbb{R})$. Let $q(\nu, a)$ be a Borel function of $(\nu, a) \in M_{p}(\mathbb{R}) \times$ $\mathbb{R}$ satisfying certain regularity conditions to be specified. A martingale problem for a continuous process $\left\{Y_{t}: t \geq 0\right\}$ in $M_{p}(\mathbb{R})$ can be formulated in the following way: For each $\phi \in C_{p}^{2}(\mathbb{R})$,

$$
M_{t}(\phi)=\left\langle\phi, Y_{t}\right\rangle-\left\langle\phi, Y_{0}\right\rangle-\frac{1}{2} \rho(0) \int_{0}^{t}\left\langle\phi^{\prime \prime}, Y_{s}\right\rangle d s-\int_{0}^{t}\left\langle q\left(Y_{s}, \cdot\right) \phi, m\right\rangle d s
$$

is a continuous martingale with quadratic variation process

$$
\langle M(\phi)\rangle_{t}=\int_{0}^{t}\left\langle\sigma \phi^{2}, Y_{s}\right\rangle d s+\int_{0}^{t} d s \int_{\mathbb{R}^{2}} \rho(x-y) \phi^{\prime}(x) \phi^{\prime}(y) Y_{s}(d x) Y_{s}(d y) .
$$

A solution $\left\{Y_{t}: t \geq 0\right\}$ of the martingale problem is called an immigration superprocess with dependent spatial motion (ISDSM); see Ref. [3]. The phrase superprocess with dependent spatial motion (SDSM) naturally refers to the special case $m=0$. Compared with the classical super Brownian motion over the real line, the last term in (1.2) represents an immigration factor with interactive immigration rate while the last term in (1.3) comes from the dependent spatial motion. In particular, if $q(\nu, a)=q(a)$ only depends on $a \in \mathbb{R}$, the immigration becomes non-interactive and the uniqueness of solution of the martingale problem can be proved by a duality argument or a conditional log-Laplace functional; see Refs. [4, 8, 10, 16]. The uniqueness of solution of the general martingale problem still remains a challenging open problem.

An ISDSM was constructed in Ref. [3] in the special case $p=0$. Instead of the martingale problem, the authors considered a stochastic integral equation driven by Poisson random measures based on the excursion law of a Feller branching diffusion. They showed 
that there is a unique strong solution of the equation which also solves (1.2) and (1.3); see also Refs. [7, 13]. In this paper we extend the result of Ref. [3] to the general state space $M_{p}(\mathbb{R})$. For the study of the ISDSM, the stochastic equation has several advantages over the martingale problem formulation. For instance, the stochastic equation provides much more information on the structures of the ISDSM than the martingale problem. As pointed out in Ref. [3], from the equation we know immediately that the ISDSM lives in the space of purely atomic measures in contrast to the classical super Brownian motion; see also Ref. [15]. With that observation, it is natural to ask whether or not the corresponding occupation time process

$$
Z_{t}:=\int_{0}^{t} Y_{s} d s, \quad t \geq 0
$$

is absolutely continuous with respect to the Lebesgue measure. We give an answer to this question as an application of the stochastic equation. We shall see that $\left\{Z_{t}: t \geq\right.$ $0\}$ is really absolutely continuous and its density field $\{z(b, t): b \in \mathbb{R}, t \geq 0\}$ can be represented in terms of stochastic integrals of the excursions with respect to the Poisson random measures and certain Brownian local times. By this representation we prove that $\{z(b, t): b \in \mathbb{R}, t \geq 0\}$ is Hölder continuous of order $\alpha$ for every $\alpha<1 / 2$. It seems difficult to establish those results from (1.2) and (1.3). The simple derivations of the results given here show the efficiency of the stochastic equation in the study of properties of the immigration superprocess. As another application of the stochastic equation, we prove two scaling limit theorems for $\left\{Y_{t}: t \geq 0\right\}$. From those theorems we derive the limit theorems for the density field $\{z(b, t): b \in \mathbb{R}, t \geq 0\}$.

\section{Preliminary results}

Let $m \in M_{p}(\mathbb{R})$ and let $(\Omega, \mathscr{F}, \mathscr{F} t, \mathbf{P})$ be a filtered standard probability space satisfying the usual hypotheses. A function $\eta(\cdot, \cdot, \cdot)$ on $[0, \infty) \times \mathbb{R} \times \Omega$ is said to be simple if it is of the form

$$
\eta(s, x, \omega)=\eta_{0}(x, \omega) 1_{\left[r_{0}, r_{1}\right]}(s)+\sum_{i=1}^{\infty} \eta_{i}(x, \omega) 1_{\left[r_{i}, r_{i+1}\right]}(s),
$$

where $0=r_{0}<r_{1}<r_{2}<\ldots$ and $\eta_{i}(\cdot, \cdot)$ is $\mathscr{B}(\mathbb{R}) \times \mathscr{F}_{r_{i}}$-measurable. Let $\mathscr{P}$ be the completion with respect to $d s m(d a) \mathbf{P}(d \omega)$ of the $\sigma$-algebra on $[0, \infty) \times \mathbb{R} \times \Omega$ generated by all simple functions. We say a function on $[0, \infty) \times \mathbb{R} \times \Omega$ is predictable if it is $\mathscr{P}$ measurable. Let $L_{+}^{2}(\mathscr{P})$ denote the set of all non-negative predictable functions $\eta(\cdot, \cdot, \cdot)$ on $[0, \infty) \times \mathbb{R} \times \Omega$ such that

$$
\int_{0}^{t} \mathbf{E}\left[\left\langle\eta(s, \cdot) \phi_{p}, m\right\rangle^{2}\right] d s<\infty, \quad t \geq 0 .
$$


Suppose that $\sigma>0$ and $\eta(\cdot, \cdot, \cdot) \in L_{+}^{2}(\mathscr{P})$. Given $\mu \in M_{p}(\mathbb{R})$, we consider the following martingale problem of a continuous process $\left\{Y_{t}: t \geq 0\right\}$ in $M_{p}(\mathbb{R})$ : For each $\phi \in C_{p}^{2}(\mathbb{R})$,

$$
M_{t}(\phi)=\left\langle\phi, Y_{t}\right\rangle-\langle\phi, \mu\rangle-\frac{1}{2} \rho(0) \int_{0}^{t}\left\langle\phi^{\prime \prime}, Y_{s}\right\rangle d s-\int_{0}^{t}\langle\eta(s, \cdot) \phi, m\rangle d s
$$

is a continuous martingale relative to $\left(\mathscr{F}_{t}\right)_{t \geq 0}$ with quadratic variation process

$$
\langle M(\phi)\rangle_{t}=\int_{0}^{t}\left\langle\sigma \phi^{2}, Y_{s}\right\rangle d s+\int_{0}^{t} d s \int_{\mathbb{R}^{2}} \rho(x-y) \phi^{\prime}(x) \phi^{\prime}(y) Y_{s}(d x) Y_{s}(d y) .
$$

A solution of (2.3) and (2.4) can be regarded as an generalized immigration superprocess with immigration rate given by the two parameter process $\{\eta(s, a): s \geq 0, a \in \mathbb{R}\}$.

Proposition 2.1 Suppose that $\left\{Y_{t}: t \geq 0\right\}$ is a solution of the martingale problem given by (2.3) and (2.4). Let $c \geq 0$ be a constant such that $\left|\phi_{p}^{\prime}\right|+\left|\phi_{p}^{\prime \prime}\right| \leq c \phi_{p}$ and let $C(t)=c^{2}\|\rho\|(16+\|\rho\| t)$. Then we have

$$
\mathbf{E}\left[\left\langle\phi_{p}, Y_{t}\right\rangle\right] \leq G_{t}\left(\phi_{p}\right)+\frac{1}{2} c\|\rho\| \int_{0}^{t} G_{s}\left(\phi_{p}\right) \exp \left\{\frac{1}{2} c\|\rho\|(t-s)\right\} d s
$$

and

$$
\mathbf{E}\left[\sup _{0 \leq s \leq t}\left\langle\phi_{p}, Y_{s}\right\rangle^{2}\right] \leq H_{t}\left(\phi_{p}\right)+C(t) \int_{0}^{t} H_{s}\left(\phi_{p}\right) \exp \{C(t)(t-s)\} d s,
$$

where

$$
G_{t}\left(\phi_{p}\right)=\left\langle\phi_{p}, \mu\right\rangle+\int_{0}^{t} \mathbf{E}\left[\left\langle\eta(s, \cdot) \phi_{p}, m\right\rangle\right] d s
$$

and

$$
H_{t}\left(\phi_{p}\right)=4\left\langle\phi_{p}, \mu\right\rangle^{2}+4 t \int_{0}^{t} \mathbf{E}\left[\left\langle\eta(s, \cdot) \phi_{p}, m\right\rangle^{2}\right] d s+16 \sigma \int_{0}^{t} \mathbf{E}\left[\left\langle\phi_{p}, Y_{s}\right\rangle\right] d s .
$$

Proof. The first inequality follows by taking the expectations in (2.3) and applying Gronwall's inequality. By (2.3) and the martingale inequality we have

$$
\begin{aligned}
\mathbf{E}\left[\sup _{0 \leq s \leq t}\left\langle\phi_{p}, Y_{s}\right\rangle^{2}\right] \leq & 4\left\langle\phi_{p}, \mu\right\rangle^{2}+4 \mathbf{E}\left[\left(\int_{0}^{t}\left\langle\eta(s, \cdot) \phi_{p}, m\right\rangle d s\right)^{2}\right] \\
& +\|\rho\|^{2} \mathbf{E}\left[\left(\int_{0}^{t}\left\langle\phi_{p}^{\prime \prime}, Y_{s}\right\rangle d s\right)^{2}\right] \\
& +16 \int_{0}^{t} \mathbf{E}\left[\sigma\left\langle\phi_{p}^{2}, Y_{s}\right\rangle+\|\rho\|\left\langle\phi_{p}^{\prime}, Y_{s}\right\rangle^{2}\right] d s \\
\leq & 4\left\langle\phi_{p}, \mu\right\rangle^{2}+4 t \int_{0}^{t} \mathbf{E}\left[\left\langle\eta(s, \cdot) \phi_{p}, m\right\rangle^{2}\right] d s \\
& +c^{2}\|\rho\|^{2} t \int_{0}^{t} \mathbf{E}\left[\left\langle\phi_{p}, Y_{s}\right\rangle^{2}\right] d s \\
& +16 \int_{0}^{t}\left(\sigma \mathbf{E}\left[\left\langle\phi_{p}, Y_{s}\right\rangle\right]+c^{2}\|\rho\| \mathbf{E}\left[\left\langle\phi_{p}, Y_{s}\right\rangle^{2}\right]\right) d s .
\end{aligned}
$$


Therefore, we can use Gronwall's inequality again to get (2.6).

Clearly, the family of martingales $\left\{M_{t}(\phi)\right\}$ in (2.3) defines a martingale measure $M(d s, d x)$ in the sense of Ref. [14]. The next result follows by standard arguments.

Proposition 2.2 Suppose that $\left\{Y_{t}: t \geq 0\right\}$ is a solution of the martingale problem given by (2.3) and (2.4). Then for any $t \geq 0$ and $\phi \in C_{p}^{2}(\mathbb{R})$ we have a.s.

$$
\left\langle\phi, Y_{t}\right\rangle=\left\langle P_{t} \phi, \mu\right\rangle+\int_{0}^{t}\left\langle\eta(s, \cdot) P_{t-s} \phi, m\right\rangle d s+\int_{0}^{t} \int_{\mathbb{R}} P_{t-s} \phi(x) M(d s, d x),
$$

where $\left(P_{t}\right)_{t \geq 0}$ is the semigroup of the Brownian motion with quadratic variation $\rho(0) d t$. Consequently,

$$
\mathbf{E}\left[\left\langle\phi, Y_{t}\right\rangle\right]=\left\langle P_{t} \phi, \mu\right\rangle+\int_{0}^{t} \mathbf{E}\left[\left\langle\eta(s, \cdot) P_{t-s} \phi, m\right\rangle\right] d s .
$$

Now we can give the construction of a solution of the martingale problem (2.3) and (2.4) with initial state $\mu \in M_{p}^{a}(\mathbb{R})$. Let $\{B(t): t \geq 0\}$ be a standard Brownian motion. For any initial condition $\xi(0)=x \geq 0$ the stochastic differential equation

$$
d \xi(t)=\sqrt{\sigma \xi(t)} d B(t), \quad t \geq 0,
$$

has a unique solution, which is known as a Feller branching diffusion with constant branching rate $\sigma$. In the sequel, we simply call $\{\xi(t): t \geq 0\}$ a $\sigma$-branching diffusion. Let $W=C\left([0, \infty), \mathbb{R}^{+}\right)$and let $\tau_{0}(w)=\inf \{s>0: w(s)=0\}$ for $w \in W$. We denote by $W_{0}$ be the set of paths $w \in W$ such that $w(t)=w(0)=0$ for every $t \geq \tau_{0}(w)$. Let $\left(\mathscr{B}\left(W_{0}\right), \mathscr{B}_{t}\left(W_{0}\right)\right)$ be the natural $\sigma$-algebras on $W_{0}$ generated by the coordinate process and let $\mathbf{Q}_{\kappa}$ be the excursion law of the $\sigma$-branching diffusion defined in Ref. [3]. Suppose that on a complete standard probability space $(\Omega, \mathscr{F}, \mathbf{P})$ the following are defined:

(2.a) a white noise $W(d s, d y)$ on $[0, \infty) \times \mathbb{R}$ based on the Lebesgue measure;

(2.b) a sequence of independent $\sigma$-branching diffusions $\left\{\xi_{i}(t): t \geq 0\right\}$ with deterministic initial values $\xi_{i}(0)=\xi_{i}, i=1,2, \cdots$;

(2.c) a Poisson random measure $N_{1}(d s, d a, d u, d w)$ on $[0, \infty) \times \mathbb{R} \times[0, \infty) \times W_{0}$ with intensity $d s m(d a) d u \mathbf{Q}_{\kappa}(d w)$.

We assume that $\{W(d s, d y)\},\left\{\xi_{i}(t)\right\}$ and $\left\{N_{1}(d s, d a, d u, d w)\right\}$ are independent of each other. For $t \geq 0$ let $\mathscr{F}_{t}$ be the $\sigma$-algebra generated by all $\mathbf{P}$-null sets and the families of random variables

$$
\left\{W([0, s] \times B), \xi_{i}(s): 0 \leq s \leq t ; B \in \mathscr{B}(\mathbb{R}), i=1,2, \cdots\right\}
$$

and

$$
\left\{N_{1}(J \times A): J \in \mathscr{B}([0, s] \times \mathbb{R} \times[0, \infty)) ; A \in \mathscr{B}_{t-s}\left(W_{0}\right) ; 0 \leq s \leq t\right\} .
$$


It is known that for any $(r, a) \in[0, \infty) \times \mathbb{R}$ there is a unique solution $\left\{x_{r, a}(t): t \geq r\right\}$ of the equation

$$
x(t)=a+\int_{r}^{t} \int_{\mathbb{R}} h(y-x(s)) W(d s, d y), \quad t \geq r
$$

see Refs. 44, 15]. Indeed, each $\left\{x_{r, a}(t): t \geq r\right\}$ is a Brownian motion with quadratic variation $\rho(0) d t$. Let $\left\{a_{i}: i=1,2, \cdots\right\} \subset \mathbb{R}$ and assume $\sum_{i=1}^{\infty} \xi_{i} \phi_{p}\left(a_{i}\right)<\infty$. For $\eta(\cdot, \cdot, \cdot) \in L_{+}^{2}(\mathscr{P})$ we define the purely atomic measure-valued process $\left\{Y_{t}: t \geq 0\right\}$ by

$$
Y_{t}=\sum_{i=1}^{\infty} \xi_{i}(t) \delta_{x_{0, a_{i}}(t)}+\int_{0}^{t} \int_{\mathbb{R}} \int_{0}^{\eta(s, a)} \int_{W_{0}} w(t-s) \delta_{x_{s, a}(t)} N_{1}(d s, d a, d u, d w) .
$$

(We make the convention that $\int_{a}^{b}=\int_{(a, b]} \cdot$ )

Theorem 2.1 The process $\left\{Y_{t}: t \geq 0\right\}$ defined by (2.13) has a continuous modification in $M_{p}^{a}(\mathbb{R})$ which solves the martingale problem given by (2.3) and (2.4).

Proof. For each integer $k \geq 1$ let $\eta_{k}(t, a)=\eta(t, a) \wedge\left(k \phi_{p}(a)\right)$. Then $t \mapsto \mathbf{E}\left[\left\langle\eta_{k}(t, \cdot), m\right\rangle^{2}\right]$ is a locally bounded function and

$$
\int_{0}^{t} \mathbf{E}\left[\left\langle\left|\eta(s, \cdot)-\eta_{k}(s, \cdot)\right| \phi_{p}, m\right\rangle^{2}\right] d s \rightarrow 0
$$

as $k \rightarrow \infty$ for every $t \geq 0$. By Theorem 5.1 of Ref. [3], the process $\left\{Y_{t}^{(k)}: t \geq 0\right\}$ defined by

$$
Y_{t}^{(k)}=\sum_{\left|a_{i}\right| \leq k} \xi_{i}(t) \delta_{x_{0, a_{i}}(t)}+\int_{0}^{t} \int_{\mathbb{R}} \int_{0}^{\eta_{k}(s, a)} \int_{W_{0}} w(t-s) \delta_{x_{s, a}(t)} N_{1}(d s, d a, d u, d w)
$$

has a continuous modification in $M_{0}^{a}(\mathbb{R})$ and hence in $M_{p}^{a}(\mathbb{R})$. Note also that $\left\{Y_{t}^{(k)}: t \geq 0\right\}$ is increasing in $k \geq 1$. For $n \geq k \geq 1$ let $g_{k, n}=\eta_{n}-\eta_{k}, \mu_{k, n}=\sum_{k<\left|a_{i}\right| \leq n} \xi_{i}(0) \delta_{a_{i}}$ and

$$
X_{t}^{(k, n)}=\sum_{k<\left|a_{i}\right| \leq n} \xi_{i}(t) \delta_{x_{0, a_{i}}(t)}+\int_{0}^{t} \int_{\mathbb{R}} \int_{\eta_{k}(s, a)}^{\eta_{n}(s, a)} \int_{W_{0}} w(t-s) \delta_{x_{s, a}(t)} N_{1}(d s, d a, d u, d w) .
$$

As in the proofs of Theorems 4.1 and 4.5 in Ref. [3] it follows that $\left\{X_{t}^{(k, n)}: t \geq 0\right\}$ has a continuous modification in $M_{p}^{a}(\mathbb{R})$ and for each $\phi \in C_{p}^{2}(\mathbb{R})$,

$$
M_{t}^{(k, n)}(\phi)=\left\langle\phi, X_{t}^{(k, n)}\right\rangle-\left\langle\phi, \mu_{k, n}\right\rangle-\int_{0}^{t}\left\langle g_{k, n}(s, \cdot) \phi, m\right\rangle d s-\frac{1}{2} \rho(0) \int_{0}^{t}\left\langle\phi^{\prime \prime}, X_{s}^{(k, n)}\right\rangle d s
$$

is a continuous martingale with quadratic variation process

$$
\int_{0}^{t}\left\langle\sigma \phi^{2}, X_{s}^{(k, n)}\right\rangle d s+\int_{0}^{t} d s \int_{\mathbb{R}^{2}} \rho(x-y) \phi^{\prime}(x) \phi^{\prime}(y) X_{s}^{(k, n)}(d x) X_{s}^{(k, n)}(d y) ;
$$


see also Lemma 3.1 of Ref. [7]. Let $C(t)$ be defined as in Proposition 2.1, We have

$$
\mathbf{E}\left[\left\langle\phi_{p}, X_{t}^{(k, n)}\right\rangle\right] \leq G_{t}^{(k, n)}\left(\phi_{p}\right)+\frac{1}{2} c\|\rho\| \int_{0}^{t} G_{s}^{(k, n)}\left(\phi_{p}\right) \exp \left\{\frac{1}{2} c\|\rho\|(t-s)\right\} d s
$$

and

$$
\mathbf{E}\left[\sup _{0 \leq s \leq t}\left\langle\phi_{p}, X_{s}^{(k, n)}\right\rangle^{2}\right] \leq H_{t}^{(k, n)}(\phi)+C(t) \int_{0}^{t} H_{s}^{(k, n)}(\phi) \exp \{C(t)(t-s)\} d s,
$$

where

$$
G_{t}^{(k, n)}\left(\phi_{p}\right)=\left\langle\phi_{p}, \mu_{k, n}\right\rangle+\int_{0}^{t} \mathbf{E}\left[\left\langle g_{k, n}(s, \cdot) \phi_{p}, m\right\rangle\right] d s
$$

and

$$
\begin{aligned}
H_{t}^{(k, n)}\left(\phi_{p}\right)= & 4\left\langle\phi_{p}, \mu_{k, n}\right\rangle^{2}+4 t \int_{0}^{t} \mathbf{E}\left[\left\langle g_{k, n}(s, \cdot) \phi_{p}, m\right\rangle^{2}\right] d s \\
& +16 \sigma \int_{0}^{t} \mathbf{E}\left[\left\langle\phi_{p}, X_{s}^{(k, n)}\right\rangle\right] d s .
\end{aligned}
$$

Clearly, we have $G_{t}^{(k, n)}\left(\phi_{p}\right) \rightarrow 0$ and $H_{t}^{(k, n)}\left(\phi_{p}\right) \rightarrow 0$ as $k, n \rightarrow \infty$. It follows that

$$
\mathbf{E}\left[\sup _{0 \leq s \leq t}\left\langle\phi_{p}, X_{s}^{(k, n)}\right\rangle^{2}\right] \rightarrow 0
$$

as $k, n \rightarrow \infty$. Then there is a continuous process $\left\{X_{t}: t \geq 0\right\}$ in $M_{p}^{a}(\mathbb{R})$ such that

$$
\mathbf{E}\left[\sup _{0 \leq s \leq t}\left\langle\phi_{p}, X_{s}-Y_{s}^{(k)}\right\rangle^{2}\right] \rightarrow 0
$$

as $n \rightarrow \infty$ for every $t \geq 0$. Clearly, $\left\{X_{t}: t \geq 0\right\}$ is independent of the particular choice of the approximating sequence $\left\{\eta_{k}\right\} \subset L_{+}^{2}(\mathscr{P})$ and the martingale characterizations (2.3) and (2.4) hold with $\left\{Y_{t}: t \geq 0\right\}$ replaced by $\left\{X_{t}: t \geq 0\right\}$. Then, to finish the proof we only need to show a.s. $X_{t}=Y_{t}$ for every $t \geq 0$. Recall that $\int_{0}^{\eta(s, a)}$ means $\int_{(0, \eta(s, a)]}$ in (2.13). Then we have a.s. $X_{t} \leq Y_{t}$ from the limit procedure for the construction of $X_{t}$. Let $\left\{X_{t}^{(k)}: t \geq 0\right\}$ be constructed by the same procedure for $\eta(\cdot, \cdot, \cdot)+1 / k \in L_{+}^{2}(\mathscr{P})$. Obviously, we have a.s. $Y_{t} \leq X_{t}^{(k)}$. However, by Proposition 2.2 we have

$$
\mathbf{E}\left[\left\langle\phi, X_{t}\right\rangle\right]=\left\langle P_{t} \phi, \mu\right\rangle+\int_{0}^{t} \mathbf{E}\left[\left\langle\eta(s, \cdot) P_{t-s} \phi, m\right\rangle\right] d s
$$

and

$$
\mathbf{E}\left[\left\langle\phi, X_{t}^{(k)}\right\rangle\right]=\left\langle P_{t} \phi, \mu\right\rangle+\int_{0}^{t} \mathbf{E}\left[\left\langle(\eta(s, \cdot)+1 / k) P_{t-s} \phi, m\right\rangle\right] d s .
$$


It follows that

$$
\mathbf{E}\left[\left\langle\phi, X_{t}\right\rangle\right] \leq \mathbf{E}\left[\left\langle\phi, Y_{t}\right\rangle\right] \leq \lim _{k \rightarrow \infty} \mathbf{E}\left[\left\langle\phi, X_{t}^{(k)}\right\rangle\right]=\mathbf{E}\left[\left\langle\phi, X_{t}\right\rangle\right]
$$

That proves $X_{t}=Y_{t}$ a.s. for every $t \geq 0$.

A solution of (2.3) and (2.4) with general initial state $\mu \in M_{p}(\mathbb{R})$ can be constructed in the following way. Let $\{W(d s, d y)\}$ and $\left\{N_{1}(d s, d a, d u, d w)\right\}$ be given as in (2.a) and (2.c) and suppose we are also given

(2.d) a Poisson random measure $N_{0}(d a, d w)$ on $\mathbb{R} \times W_{0}$ with intensity $\mu(d a) \mathbf{Q}_{\kappa}(d w)$.

We assume that $\{W(d s, d y)\},\left\{N_{0}(d a, d w)\right\}$ and $\left\{N_{1}(d s, d a, d u, d w)\right\}$ are independent of each other. For $t \geq 0$ let $\mathscr{F}_{t}$ be the $\sigma$-algebra generated by all $\mathbf{P}$-null sets and the families of random variables

$$
\left\{W([0, s] \times B), N_{0}(F \times A): 0 \leq s \leq t, B \in \mathscr{B}(\mathbb{R}), A \in \mathscr{B}_{t}\left(W_{0}\right)\right\}
$$

and

$$
\left\{N_{1}(J \times A): J \in \mathscr{B}([0, s] \times \mathbb{R} \times[0, \infty)), A \in \mathscr{B}_{t-s}\left(W_{0}\right), 0 \leq s \leq t\right\} .
$$

Let $\left\{X_{t}: t \geq 0\right\}$ be defined by $X_{0}=\mu$ and

$$
X_{t}=\int_{\mathbb{R}} \int_{W_{0}} w(t) \delta_{x_{0, a}(t)} N_{0}(d a, d w), \quad t>0 .
$$

For $\eta(\cdot, \cdot, \cdot) \in L_{+}^{2}(\mathscr{P})$ we define

$$
Y_{t}=X_{t}+\int_{0}^{t} \int_{\mathbb{R}} \int_{0}^{\eta(s, a)} \int_{W_{0}} w(t-s) \delta_{x_{s, a}(t)} N_{1}(d s, d a, d u, d w), \quad t \geq 0 .
$$

By similar arguments as in the proof of Theorem 2.1 we obtain

Theorem 2.2 The process $\left\{Y_{t}: t \geq 0\right\}$ defined by (2.17) has a continuous modification in $M_{p}(\mathbb{R})$ which solves the martingale problem given by (2.3) and (2.4).

\section{Stochastic equations for superprocesses}

In this section, we give the construction of the ISDSM by solving stochastic equations driven by Poisson random measures on the space of excursions. For any $\mu$ and $\nu \in M_{p}(\mathbb{R})$ set

$$
\|\mu-\nu\|_{p}=\sup _{f \in B_{1}(\mathbb{R})}\left|\int_{\mathbb{R}} f(x) \phi_{p}(x) \mu(d x)-\int_{\mathbb{R}} f(x) \phi_{p}(x) \nu(d x)\right|,
$$


where $B_{1}(\mathbb{R})$ denotes the set of Borel functions $f$ on $\mathbb{R}$ such that $|f(x)| \leq 1$ for all $x \in \mathbb{R}$. Suppose that $q(\cdot, \cdot)$ is a Borel function on $M_{p}(\mathbb{R}) \times \mathbb{R}$ such that there is a constant $K$ such that

$$
\left\langle q(\mu, \cdot) \phi_{p}, m\right\rangle \leq K\left(1+\left\langle\phi_{p}, \mu\right\rangle\right), \quad \mu \in M(\mathbb{R}),
$$

and for each $R>0$ there is a constant $L_{R}>0$ such that

$$
\left\langle|q(\mu, \cdot)-q(\nu, \cdot)| \phi_{p}, m\right\rangle \leq L_{R}\|\mu-\nu\|_{p}
$$

for $\mu$ and $\nu \in M_{p}(\mathbb{R})$ satisfying $\left\langle\phi_{p}, \mu\right\rangle \leq R$ and $\left\langle\phi_{p}, \nu\right\rangle \leq R$. Given $\mu \in M_{p}(\mathbb{R})$, we consider the following martingale problem of a continuous process $\left\{Y_{t}: t \geq 0\right\}$ in $M_{p}(\mathbb{R})$ : For each $\phi \in C_{p}^{2}(\mathbb{R})$,

$$
M_{t}(\phi)=\left\langle\phi, Y_{t}\right\rangle-\langle\phi, \mu\rangle-\frac{1}{2} \rho(0) \int_{0}^{t}\left\langle\phi^{\prime \prime}, Y_{s}\right\rangle d s-\int_{0}^{t}\left\langle q\left(Y_{s}, \cdot\right) \phi, m\right\rangle d s
$$

is a continuous martingale with quadratic variation process

$$
\langle M(\phi)\rangle_{t}=\int_{0}^{t}\left\langle\sigma \phi^{2}, Y_{s}\right\rangle d s+\int_{0}^{t} d s \int_{\mathbb{R}^{2}} \rho(x-y) \phi^{\prime}(x) \phi^{\prime}(y) Y_{s}(d x) Y_{s}(d y) .
$$

By similar arguments as in the proof of Proposition 2.1 we have the following

Proposition 3.1 Suppose that $\left\{Y_{t}: t \geq 0\right\}$ is a solution of the martingale problem given by (3.4) and (3.5). Let $c \geq 0$ be a constant such that $\left|\phi_{p}^{\prime}\right|+\left|\phi_{p}^{\prime \prime}\right| \leq c \phi_{p}$ and let $C_{1}=K+c\|\rho\| / 2$ and $C_{2}(t)=c^{2}\|\rho\|(16+\|\rho\| t)$. Then we have

$$
\mathbf{E}\left[\left\langle\phi_{p}, Y_{t}\right\rangle\right] \leq G_{t}\left(\phi_{p}\right)+C_{1} \int_{0}^{t} G_{s}\left(\phi_{p}\right) \exp \left\{C_{1}(t-s)\right\} d s
$$

and

$$
\mathbf{E}\left[\sup _{0 \leq s \leq t}\left\langle\phi_{p}, Y_{s}\right\rangle^{2}\right] \leq H_{t}\left(\phi_{p}\right)+C_{2}(t) \int_{0}^{t} H_{s}\left(\phi_{p}\right) \exp \left\{C_{2}(t)(t-s)\right\} d s,
$$

where $G_{t}\left(\phi_{p}\right)=\left\langle\phi_{p}, \mu\right\rangle+K t$ and

$$
H_{t}\left(\phi_{p}\right)=4\left\langle\phi_{p}, \mu\right\rangle^{2}+4 K^{2} t^{2}+8(K t+2 \sigma) \int_{0}^{t} \mathbf{E}\left[\left\langle\phi_{p}, Y_{s}\right\rangle\right] d s .
$$

Let $\{W(d s, d y)\},\left\{\xi_{i}(t)\right\}$ and $\left\{N_{1}(d s, d a, d u, d w)\right\}$ be given as in (2.a), (2.b) and (2.c). Let $\left(\mathscr{F}_{t}\right)_{t \geq 0}$ be the filtration generated by (2.10) and (2.11). By a solution of the stochastic equation

$$
Y_{t}=\sum_{i=1}^{\infty} \xi_{i}(t) \delta_{x_{0, a_{i}}(t)}+\int_{0}^{t} \int_{\mathbb{R}} \int_{0}^{q\left(Y_{s}, a\right)} \int_{W_{0}} w(t-s) \delta_{x_{s, a}(t)} N_{1}(d s, d a, d u, d w),
$$

we mean an $\left(\mathscr{F}_{t}\right)$-adapted continuous process $\left\{Y_{t}: t \geq 0\right\}$ in $M_{p}^{a}(\mathbb{R})$ that satisfies (3.8) for all $t \geq 0$. 
Theorem 3.1 There is an unique solution $\left\{Y_{t}: t \geq 0\right\}$ of (3.8), which is a strong Markov process in $M_{p}^{a}(\mathbb{R})$ and solves the martingale problem given by (3.4) and (3.5).

Proof. Suppose that $\left\{Y_{t}: t \geq 0\right\}$ and $\left\{Y_{t}^{\prime}: t \geq 0\right\}$ are two continuous solutions of (3.8). Fix $R \geq 1$ and let $\tau=\inf \left\{t \geq 0:\left\langle\phi_{p}, Y_{t}\right\rangle \geq R\right.$ or $\left.\left\langle\phi_{p}, Y_{t}^{\prime}\right\rangle \geq R\right\}$. Observe that $q\left(Y_{t}, x\right) 1_{\{t \leq \tau\}}$ and $q\left(Y_{t}^{\prime}, x\right) 1_{\{t \leq \tau\}}$ are predictable and define

$$
X_{t}^{*}=\int_{0}^{t \wedge \tau} \int_{\mathbb{R}} \int_{0}^{q\left(Y_{s}, a\right) \wedge q\left(Y_{s}^{\prime}, a\right)} \int_{W_{0}} w(t-s) \delta_{x_{s, a}(t)} N_{1}(d s, d a, d u, d w)
$$

and

$$
Y_{t}^{*}=\int_{0}^{t \wedge \tau} \int_{\mathbb{R}} \int_{0}^{q\left(Y_{s}, a\right) \vee q\left(Y_{s}^{\prime}, a\right)} \int_{W_{0}} w(t-s) \delta_{x_{s, a}(t)} N_{1}(d s, d a, d u, d w) .
$$

By Proposition 2.2 we have

$$
\mathbf{E}\left[\left\langle\phi, X_{t}^{*}\right\rangle\right]=\int_{0}^{t} \mathbf{E}\left[\left\langle\left(q\left(Y_{s}, \cdot\right) \wedge q\left(Y_{s}^{\prime}, \cdot\right)\right) P_{t-s} \phi, m\right\rangle 1_{\{s \leq \tau\}}\right] d s
$$

and

$$
\mathbf{E}\left[\left\langle\phi, Y_{t}^{*}\right\rangle\right]=\int_{0}^{t} \mathbf{E}\left[\left\langle\left(q\left(Y_{s}, \cdot\right) \vee q\left(Y_{s}^{\prime}, \cdot\right)\right) P_{t-s} \phi, m\right\rangle 1_{\{s \leq \tau\}}\right] d s .
$$

Let $c \geq 0$ be a constant such that $\left|\phi_{p}^{\prime \prime}\right| \leq 2 c \phi_{p}$. Then we have

$$
\frac{d}{d t} P_{t} \phi_{p}(x)=\frac{1}{2} P_{t} \phi_{p}^{\prime \prime}(x) \leq c P_{t} \phi_{p}(x) .
$$

By a comparison theorem, we get $P_{t} \phi_{p} \leq e^{c t} \phi_{p}$ for every $t \geq 0$. Observe also that $\left\|Y_{t \wedge \tau}^{\prime}-Y_{t \wedge \tau}\right\|_{p} \leq\left\langle\phi_{p}, Y_{t}^{*}\right\rangle-\left\langle\phi_{p}, X_{t}^{*}\right\rangle$. Then (3.9) and (3.10) imply that

$$
\begin{aligned}
\mathbf{E}\left[\left\|Y_{t \wedge \tau}^{\prime}-Y_{t \wedge \tau}\right\|_{p}\right] & =\int_{0}^{t} \mathbf{E}\left[\left\langle\left|q\left(Y_{s}^{\prime}, \cdot\right)-q\left(Y_{s}, \cdot\right)\right| P_{t-s} \phi_{p}, m\right\rangle 1_{\{s \leq \tau\}}\right] d s \\
& \leq e^{c t} \int_{0}^{t} \mathbf{E}\left[\left\langle\left|q\left(Y_{s}^{\prime}, \cdot\right)-q\left(Y_{s}, \cdot\right)\right| \phi_{p}, m\right\rangle 1_{\{s \leq \tau\}}\right] d s \\
& \leq L_{R} e^{c t} \int_{0}^{t} \mathbf{E}\left[\left\|Y_{s \wedge \tau}^{\prime}-Y_{s \wedge \tau}\right\| \|_{p}\right] d s .
\end{aligned}
$$

By Gronwall's inequality we conclude $\mathbf{E}\left[\left\|Y_{t \wedge \tau}^{\prime}-Y_{t \wedge \tau}\right\|_{p}\right]=0$. Since $R \geq 0$ can be arbitrary, that gives the uniqueness of solution. To show the existence of a solution, we first assume (3.3) holds with $L_{R}$ replaced by a universal constant $L$ independent of $R$. We define the sequence continuous processes $\left\{Y_{t}^{(n)}: t \geq 0\right\}$ by setting $Y_{t}^{(0)}=\sum_{i=1}^{\infty} \xi_{i}(t) \delta_{x_{0, a_{i}}(t)}$ and

$$
Y_{t}^{(n)}=Y_{t}^{(0)}+\int_{0}^{t} \int_{\mathbb{R}} \int_{0}^{q\left(Y_{s}^{(n-1)}, a\right)} \int_{W_{0}} w(t-s) \delta_{x_{s, a}(t)} N_{1}(d s, d a, d u, d w)
$$


for $n \geq 1$. By a reasoning as in (3.11) we see that

$$
\mathbf{E}\left[\left\|Y_{t}^{(n)}-Y_{t}^{(n-1)}\right\|_{p}\right] \leq L e^{c t} \int_{0}^{t} \mathbf{E}\left[\left\|Y_{s}^{(n-1)}-Y_{s}^{(n-2)}\right\|_{p}\right] d s .
$$

By a standard argument, one sees there is a predictable process $\left\{Y_{t}: t \geq 0\right\}$ such that $\lim _{n \rightarrow \infty} \mathbf{E}\left[\left\|Y_{t}-Y_{t}^{(n)}\right\|_{p}\right]=0$ uniformly on each bounded interval of $t \geq 0$. By Theorem 2.1 we see that

$$
\tilde{Y}_{t}=Y_{t}^{(0)}+\int_{0}^{t} \int_{\mathbb{R}} \int_{0}^{q\left(Y_{s}, a\right)} \int_{W_{0}} w(t-s) \delta_{x_{s, a}(t)} N_{1}(d s, d a, d u, d w)
$$

defines a continuous process $\left\{\tilde{Y}_{t}: t \geq 0\right\}$ on $M_{p}(\mathbb{R})$. Based on (3.12) and (3.13) we may follow the calculations in (3.11) to obtain

$$
\mathbf{E}\left[\left\|Y_{t}^{(n)}-\tilde{Y}_{t}\right\|_{p}\right] \leq L e^{c t} \int_{0}^{t} \mathbf{E}\left[\left\|Y_{s}^{(n-1)}-Y_{s}\right\|_{p}\right] d s
$$

Letting $n \rightarrow \infty$ we see that $Y_{t}=\tilde{Y}_{t}$ a.s. for every $t \geq 0$. That proves the existence of a solution of (3.8) for a universal constant $L$. The extension of the existence to the general condition (3.3) is a standard localization argument. The strong Markov property of $\left\{Y_{t}: t \geq 0\right\}$ follows from the uniqueness of the solution.

To consider a general initial value $\mu \in M_{p}^{a}(\mathbb{R})$ let $\{W(d s, d y)\},\left\{N_{0}(d a, d w)\right\}$ and $\left\{N_{1}(d s, d a, d u, d w)\right\}$ be given as in (2.a), (2.c) and (2.d). Let $\left(\mathscr{F}_{t}\right)_{t \geq 0}$ be the filtration generated by (2.14) and (2.15). Let $\left\{X_{t}: t \geq 0\right\}$ be given by (2.16). By a solution of the stochastic equation

$$
Y_{t}=X_{t}+\int_{0}^{t} \int_{\mathbb{R}} \int_{0}^{q\left(Y_{s}, a\right)} \int_{W_{0}} w(t-s) \delta_{x_{s, a}(t)} N_{1}(d s, d a, d u, d w), \quad t \geq 0
$$

we mean an $\left(\mathscr{F}_{t}\right)$-adapted continuous process $\left\{Y_{t}: t \geq 0\right\}$ in $M_{p}(\mathbb{R})$ that satisfies (3.14). By arguments similar to those in the proof of Theorem 3.1 we have

Theorem 3.2 There is a unique solution $\left\{Y_{t}: t \geq 0\right\}$ of (3.14), which also solves the martingale problem given by (3.4) and (3.5).

\section{Existence and continuity of local times}

In this section, we prove that the occupation time of an immigration superprocess $\left\{Y_{t}\right.$ : $t \geq 0\}$ has a Hölder continuous density field $\{z(b, t): b \in \mathbb{R}, t \geq 0\}$. As in the classical case the two parameter process $\{z(b, t): b \in \mathbb{R}, t \geq 0\}$ can be interpreted as the local time of $\left\{Y_{t}: t \geq 0\right\}$. We shall need the following two lemmas. 
Lemma 4.1 Let $\left\{B_{j}(\cdot): j=1, \cdots, n\right\}$ be a family of Brownian motions, $\left\{\xi_{j}(\cdot): j=\right.$ $1, \cdots, n\}$ be a family of independent $\sigma$-branching diffusions and $\left\{\alpha_{j}(\cdot): j=1, \cdots, n\right\}$ be a family of bounded processes. Suppose that the two families $\left\{B_{j}(\cdot): j=1, \cdots, n\right\}$ and $\left\{\xi_{j}(\cdot): j=1, \cdots, n\right\}$ are independent of each other. Let $l_{j}(\cdot, \cdot)$ denote the local time of $B_{j}(\cdot)$. Then to each integer $k \geq 1$ there corresponds a constant $C_{k} \geq 0$ such that

$$
\begin{aligned}
\mathbf{E}\{[ & {\left.\left[\sum_{j=1}^{n} \int_{r_{j}}^{t_{j}} \xi_{j}(s) \alpha_{j}(s) l_{j}(b, d s)\right]^{2 k}\right\} } \\
& \leq C_{k}(t-r)^{k}\left\{\mathbf{E}\left[\left(\sum_{j=1}^{n} \xi_{j}\left(t_{j}\right)\right)^{2 k}\right]+\mathbf{E}\left[\left(\sum_{j=1}^{n} \int_{r_{j}}^{t_{j}} \xi_{j}(s) d s\right)^{k}\right]\right\}
\end{aligned}
$$

for any intervals $\left[r_{j}, t_{j}\right] \subset[r, t], j=1, \cdots, n$.

Proof. In this and the following proofs, $C_{k}$ will denote positive constants that may change values from line to line. By integration by parts formula, we have

$$
\begin{aligned}
\int_{r_{j}}^{t_{j}} \xi_{j}(s) \alpha_{j}(s) l_{j}(b, d s)=\xi_{j}\left(t_{j}\right) & \int_{r_{j}}^{t_{j}} \alpha_{j}(s) l_{j}(b, d s) \\
& -\int_{r_{j}}^{t_{j}} d \xi_{j}(s) \int_{r_{j}}^{s} \alpha_{j}(s) l_{j}(b, d s) .
\end{aligned}
$$

By Hölder's inequality and the boundedness of $\left\{\alpha_{j}(\cdot): j=1, \cdots, n\right\}$,

$$
\begin{aligned}
\mathbf{E}\left\{\left[\sum_{j=1}^{n} \xi_{j}\left(t_{j}\right) \int_{r_{j}}^{t_{j}} \alpha_{j}(s) l_{j}(b, d s)\right]^{2 k}\right\} \\
\quad \leq \mathbf{E}\left\{\left[\sum_{j=1}^{n} \xi_{j}\left(t_{j}\right)\right]^{2 k-1} \sum_{j=1}^{n} \xi_{j}\left(t_{j}\right)\left[\int_{r_{j}}^{t_{j}} \alpha_{j}(s) l_{j}(b, d s)\right]^{2 k}\right\} \\
\quad \leq C_{k} \mathbf{E}\left\{\left[\sum_{j=1}^{n} \xi_{j}\left(t_{j}\right)\right]^{2 k-1} \sum_{j=1}^{n} \xi_{j}\left(t_{j}\right) l_{j}\left(b,\left[r_{j}, t_{j}\right]\right)^{2 k}\right\} \\
\leq C_{k}(t-r)^{k} \mathbf{E}\left\{\left[\sum_{j=1}^{n} \xi_{j}\left(t_{j}\right)\right]^{2 k}\right\},
\end{aligned}
$$

where we also used the estimate $\mathbf{E}\left[l_{j}\left(b,\left[r_{j}, t_{j}\right]\right)^{2 k}\right] \leq C_{k}\left(t_{j}-r_{j}\right)^{k}$ and the independence of $\xi_{j}(\cdot)$ and $l_{j}(\cdot, \cdot)$ for the last inequality. Similarly, by Doob's martingale inequality we get

$$
\begin{aligned}
\mathbf{E}\left\{\left[\sum_{j=1}^{n} \int_{r_{j}}^{t_{j}} d \xi_{j}(s) \int_{r_{j}}^{s} \alpha_{j}(s) l_{j}(b, d s)\right]^{2 k}\right\} \\
\quad \leq C_{k} \mathbf{E}\left\{\left[\sum_{j=1}^{n} \int_{r_{j}}^{t_{j}}\left(\int_{r_{j}}^{s} \alpha_{j}(s) l_{j}(b, d s)\right)^{2} \xi_{j}(s) d s\right]^{k}\right\}
\end{aligned}
$$




$$
\begin{aligned}
& \leq C_{k} \mathbf{E}\left\{\left[\sum_{j=1}^{n} \int_{r_{j}}^{t_{j}} l_{j}\left(b,\left[r_{j}, s\right]\right)^{2} \xi_{j}(s) d s\right]^{k}\right\} \\
& \leq C_{k} \mathbf{E}\left\{\left[\sum_{j=1}^{n} \int_{r_{j}}^{t_{j}} \xi_{j}(s) d s\right]^{k-1} \sum_{j=1}^{n} \int_{r_{j}}^{t_{j}} l_{j}\left(b,\left[r_{j}, s\right]\right)^{2 k} \xi_{j}(s) d s\right\} \\
& \leq C_{k}(t-r)^{k} \mathbf{E}\left\{\left[\sum_{j=1}^{n} \int_{r_{j}}^{t_{j}} \xi_{j}(s) d s\right]^{k}\right\} .
\end{aligned}
$$

Then the desired estimate follows from (4.2), (4.3) and (4.4).

Lemma 4.2 Under the assumptions of Lemma 4.1, to each integer $k \geq 1$ there corresponds a constant $C_{k} \geq 0$ such that

$$
\begin{aligned}
\mathbf{E}\{\mid & \left.\left.\sum_{j=1}^{n}\left[\int_{r_{j}}^{t_{j}} \xi_{j}(s) \alpha_{j}(s) l_{j}\left(b_{1}, d s\right)-\int_{r_{j}}^{t_{j}} \xi_{j}(s) \alpha_{j}(s) l_{j}\left(b_{2}, d s\right)\right]\right|^{2 k}\right\} \\
& \leq C_{k}\left(b_{1}-b_{2}\right)^{k}\left\{\mathbf{E}\left[\left(\sum_{j=1}^{n} \xi_{j}\left(t_{j}\right)\right)^{2 k}\right]+\mathbf{E}\left[\left(\sum_{j=1}^{n} \int_{r_{j}}^{t_{j}} \xi_{j}(s) d s\right)^{k}\right]\right\}
\end{aligned}
$$

for any $b_{1}, b_{2} \in \mathbb{R}$ and any intervals $\left[r_{j}, t_{j}\right] \subset[r, t], j=1, \cdots, n$.

Proof. By a formula at page 211 of Ref. [12] it is easy to show that

$$
\mathbf{E}\left\{\left[l_{j}\left(b_{1},\left[r_{j}, t_{j}\right]\right)-l_{j}\left(b_{2},\left[r_{j}, t_{j}\right]\right)\right]^{2 k}\right\} \leq C_{k}\left(b_{1}-b_{2}\right)^{k} .
$$

We can apply (4.2) to the two integrals on the left hand side of (4.5). Then the result is obtained by similar estimates as in the proof of Lemma 4.1 .

Theorem 4.1 Let $\eta(\cdot, \cdot, \cdot) \in L_{+}^{2}(\mathscr{P})$ and suppose there is a deterministic increasing function $\bar{\eta}(\cdot)$ on $[0, \infty)$ such that $\eta(t, a, \omega) \leq \bar{\eta}(t)$ for all $(t, a, \omega)$. Let $\left\{Z_{t}: t \geq 0\right\}$ denote the occupation time of the process $\left\{Y_{t}: t \geq 0\right\}$ defined by (2.17). Then $Z_{t}$ is a.s. absolutely continuous and the corresponding local time is given by

$$
\begin{aligned}
z(b, t)= & \int_{\mathbb{R}} \int_{W_{0}} N_{0}(d a, d w) \int_{0}^{t} w(u) l_{0, a}(b, d u) \\
& +\int_{0}^{t} \int_{\mathbb{R}} \int_{0}^{\eta(s, a)} \int_{W_{0}} N_{1}(d s, d a, d u, d w) \int_{s}^{t} w(v-s) l_{s, a}(b, d v),
\end{aligned}
$$

where $\left\{l_{s, a}(b, u): u \geq s\right\}$ is the local time of $\left\{x_{s, a}(u): u \geq s\right\}$ at $b \in \mathbb{R}$. Moreover, the two parameter process $\{z(b, t): b \in \mathbb{R}, t \geq 0\}$ has a version which is Hölder continuous of order $\alpha$ for every $\alpha<1 / 2$. 
Proof. The existence of the local time follows by (2.17) and Fubini's theorem. Let $z_{0}(b, t)$ and $z_{1}(b, t)$ denote respectively the first and the second terms on the right hand side of (4.6). We shall only give the proof of the continuity result for $z_{1}(\cdot, \cdot)$ since the proof for $z_{0}(\cdot, \cdot)$ is similar.

Step 1. Let us consider the special case with $\mu$ and $m \in M(\mathbb{R})$. Observe that $z_{1}(b, 0)=$ 0 for every $b \in \mathbb{R}$. For any $t>r>0$ and $b \in \mathbb{R}$ we have

$$
\begin{aligned}
z_{1}(b, t)-z_{1}(b, r)= & \int_{r}^{t} \int_{\mathbb{R}} \int_{0}^{\eta(s, a)} \int_{W_{0}} N_{1}(d s, d a, d u, d w) \int_{s}^{t} w(v-s) l_{s, a}(b, d v) \\
& +\int_{0}^{r} \int_{\mathbb{R}} \int_{0}^{\eta(s, a)} \int_{W_{0}} N_{1}(d s, d a, d u, d w) \int_{r}^{t} w(v-s) l_{s, a}(b, d v) .
\end{aligned}
$$

For $\epsilon>0$ let $W_{\epsilon}=\left\{w \in W_{0}: w(\epsilon)>0\right\}$. Then $N_{1}\left([0, t] \times \mathbb{R} \times[0, \bar{\eta}(t)] \times W_{\epsilon}\right)$ is a.s. finite for every $t \geq 0$. Let $S\left(\bar{q}, N_{1}\right)$ denote the intersection of the support of $N_{1}(d s, d a, d u, d w)$ with the set $\left\{(s, a, u, w): s \geq 0, a \in \mathbb{R}, 0 \leq u \leq \bar{\eta}(s), w \in W_{\epsilon}\right\}$. We can enumerate $S\left(\bar{q}, N_{1}\right)$ into a sequence $\left\{\left(s_{j}, a_{j}, u_{j}, w_{j}\right): j=1,2, \cdots\right\}$ so that $0<s_{1}<s_{2}<\cdots$. As in the proof of Lemma 3.2 in Ref. [3] one sees that, given $\left\{\left(s_{j}, a_{j}, u_{j}, w_{j}(\epsilon)\right): j=1,2, \cdots\right\}$ each $\left\{w_{j}(u): u \geq \epsilon\right\}$ is a $\sigma$-branching diffusion independent of the white noise $\{W(d s, d y)\}$. For any integer $k \geq 1$ we may use Lemma 4.1 and Fatou's lemma to see that

$$
\begin{aligned}
& \mathbf{E}\left\{\left[\int_{r}^{t} \int_{\mathbb{R}} \int_{0}^{\eta(s, a)} \int_{W_{0}} N_{1}(d s, d a, d u, d w) \int_{s}^{t} w(v-s) l_{s, a}(b, d v)\right]^{2 k}\right\} \\
& \leq \liminf _{\epsilon \rightarrow 0} \mathbf{E}\left\{\left[\sum_{r<s_{j} \leq t} \int_{s_{j}+\epsilon}^{t} w_{j}\left(v-s_{j}\right) l_{s_{j}, a_{j}}(b, d v)\right]^{2 k}\right\} \\
& \leq C_{k}(t-r)^{k} \liminf _{\epsilon \rightarrow 0}\left\{\mathbf{E}\left[\left(\sum_{r<s_{j} \leq t} w_{j}\left(t-s_{j}\right)\right)^{2 k}\right]\right. \\
& \left.\quad+\mathbf{E}\left[\left(\sum_{r<s_{j} \leq t} \int_{\epsilon+s_{j}}^{t} w_{j}\left(v-s_{j}\right) d v\right)^{k}\right]\right\} \\
& \leq C_{k}(t-r)^{k}\left\{\mathbf{E}\left[\left(\int_{r}^{t} \int_{\mathbb{R}} \int_{0}^{\bar{\eta}(t)} \int_{W_{0}} w(t-s) N_{1}(d s, d a, d u, d w)\right)^{2 k}\right]\right. \\
& \left.\quad+\mathbf{E}\left[\left(\int_{r}^{t} \int_{\mathbb{R}} \int_{W_{0}} \int_{0}^{\bar{\eta}(t)} N_{1}(d s, d a, d u, d w) \int_{s}^{t} w(v-s) d v\right)^{k}\right]\right\} \\
& \leq C_{k}(t-r)^{k}\left\{\mathbf{E}\left[\left\langle 1, \bar{Y}_{t}\right\rangle^{2 k}\right]+\mathbf{E}\left[\left\langle 1, \bar{Z}_{t}\right\rangle^{k}\right]\right\},
\end{aligned}
$$

where $\left\{\bar{Y}_{t}\right\}$ is the process defined by (2.17) from $\{\bar{\eta}(t)\}$, and $\left\{\bar{Z}_{t}\right\}$ is the corresponding occupation time process. By a similar reasoning as the above we have

$$
\begin{gathered}
\mathbf{E}\left\{\left[\int_{0}^{r} \int_{\mathbb{R}} \int_{0}^{\eta(s, a)} \int_{W_{0}} N_{1}(d s, d a, d u, d w) \int_{r}^{t} w(u-s) l_{s, a}(b, d u)\right]^{2 k}\right\} \\
\leq \liminf _{\epsilon \rightarrow 0} \mathbf{E}\left\{\left[\sum_{0<s_{j} \leq r} \int_{r \vee\left(\epsilon+s_{j}\right)}^{t} w_{j}\left(u-s_{j}\right) l_{s_{j}, a_{j}}(b, d u)\right]^{2 k}\right\}
\end{gathered}
$$




$$
\begin{aligned}
& \leq C_{k}(t-r)^{k} \liminf _{\epsilon \rightarrow 0}\left\{\mathbf{E}\left[\left(\sum_{0<s_{j} \leq r} w_{j}\left(t-s_{j}\right)\right)^{2 k}\right]\right. \\
& \left.+\mathbf{E}\left[\left(\sum_{0<s_{j} \leq r} \int_{r \vee\left(\epsilon+s_{j}\right)}^{t} w_{j}\left(u-s_{j}\right) d u\right)^{k}\right]\right\} \\
& \leq C_{k}(t-r)^{k}\left\{\mathbf{E}\left[\left(\int_{0}^{r} \int_{\mathbb{R}} \int_{0}^{\bar{\eta}(t)} \int_{W_{0}} w(t-s) N_{1}(d s, d a, d u, d w)\right)^{2 k}\right]\right. \\
& \left.+\mathbf{E}\left[\left(\int_{0}^{r} \int_{\mathbb{R}} \int_{0}^{\bar{\eta}(t)} \int_{W_{0}} N_{1}(d s, d a, d u, d w) \int_{r}^{t} w(v-s) d v\right)^{k}\right]\right\} \\
& \leq C_{k}(t-r)^{k}\left\{\mathbf{E}\left[\left\langle 1, \bar{Y}_{t}\right\rangle^{2 k}\right]+\mathbf{E}\left[\left\langle 1, \bar{Z}_{t}\right\rangle^{k}\right]\right\} .
\end{aligned}
$$

Thus we have

$$
\mathbf{E}\left[\left|z_{1}(b, t)-z_{1}(b, r)\right|^{2 k}\right] \leq C_{k}(t-r)^{k}\left\{\mathbf{E}\left[\left\langle 1, \bar{Y}_{t}\right\rangle^{2 k}\right]+\mathbf{E}\left[\left\langle 1, \bar{Z}_{t}\right\rangle^{k}\right]\right\} .
$$

For $t>0$ and $b_{1}, b_{2} \in \mathbb{R}$ we can use Lemma 4.2 and similar arguments as the above to show

$$
\mathbf{E}\left[\left|z_{1}\left(b_{1}, t\right)-z_{1}\left(b_{2}, t\right)\right|^{2 k}\right] \leq C_{k}\left(b_{1}-b_{2}\right)^{k}\left\{\mathbf{E}\left[\left\langle 1, \bar{Y}_{t}\right\rangle^{2 k}\right]+\mathbf{E}\left[\left\langle 1, \bar{Z}_{t}\right\rangle^{k}\right]\right\} .
$$

Since $\mu$ and $m \in M(\mathbb{R})$ are finite measures and $\bar{\eta}(t)$ is a locally bounded function of $t \geq 0$, it is not hard to show that $\mathbf{E}\left[\left\langle 1, \bar{Y}_{s}\right\rangle^{2 k}+\left\langle 1, \bar{Z}_{s}\right\rangle^{k}\right]$ is locally bounded in $t \geq 0$. Then (4.7) and (4.8) imply that $z_{1}(\cdot, \cdot)$ has a Hölder continuous version of order $\alpha$ for every $\alpha<1 / 2$; see e.g. page 273 of Ref. [14].

Step 2. Now we consider the general case with $\mu$ and $m \in M_{p}(\mathbb{R})$. Let $L$ and $T$ be fixed positive constants. For any integer $n \geq 1$ let $a_{n}>0$ be sufficiently large so that

$$
\mathbf{P}\left\{\sup _{0 \leq s \leq T}\left|x_{0,0}(s)\right| \geq a_{n}\right\} \leq \frac{1}{n}
$$

Let $b_{n}=L+a_{n}$ and $c_{n}=L+2 a_{n}$. Let $A_{n}=\left\{\right.$ there exists $0 \leq s \leq T$ such that $x_{0, b_{n}}(s)=L$ or $\left.=c_{n}\right\}$ and $B_{n}=\left\{\right.$ there exists $0 \leq s \leq T$ such that $x_{0,-b_{n}}(s)=-L$ or $\left.=-c_{n}\right\}$. As observed in Ref. [16], any two solutions of (2.12) started from different locations never collide. Then on the event $\left(A_{n} \cup B_{n}\right)^{c}$, for any $|a| \geq c_{n}$ and $0 \leq s \leq u \leq T$ we have $x_{s, a}(u)>x_{0, b_{n}}(u)>L$ or $x_{s, a}(u)<x_{0,-b_{n}}(u)<-L$, and so $l_{s, a}(b, u)=0$ whenever $|b| \leq L$. It follows that

$$
\begin{aligned}
z(b, t)= & \int_{\left[-c_{n}, c_{n}\right]} \int_{W_{0}} N_{0}(d a, d w) \int_{0}^{t} w(u) l_{0, a}(b, d u) \\
& +\int_{0}^{t} \int_{\left[-c_{n}, c_{n}\right]} \int_{0}^{\eta(s, a)} \int_{W_{0}} N_{1}(d s, d a, d u, d w) \int_{s}^{t} w(v-s) l_{s, a}(b, d v)
\end{aligned}
$$

for $(b, t) \in[-L, L] \times[0, T]$ on $\left(A_{n} \cup B_{n}\right)^{c}$. By Step 1 , for any $\alpha<1 / 2$ we have a modification of $z(b, t)$ on $\left(A_{n} \cup B_{n}\right)^{c}$ that is Hölder continuous of order $\alpha$ in $(b, t) \in(-L, L) \times[0, T)$. 
In view of (4.9) we have $\mathbf{P}\left(A_{n}\right)=\mathbf{P}\left(B_{n}\right)=1 / n$. Then we can modify $z(b, t)$ on the whole space $\Omega$ so the process becomes Hölder continuous of order $\alpha$ in $(b, t) \in(-L, L) \times[0, T)$. Since $L \geq 0$ and $T \geq 0$ can be arbitrary, we have the desired result.

Theorem 4.2 Let $q(\cdot, \cdot)$ be a Borel function on $M_{p}(\mathbb{R}) \times \mathbb{R}$ satisfying the conditions in the last section. In stead of (3.2), we assume the stronger condition

$$
q(\mu, a) \leq K\left(1+\left\langle\phi_{p}, \mu\right\rangle^{2}\right), \quad a \in \mathbb{R}, \mu \in M_{p}(\mathbb{R}) .
$$

Let $\left\{Z_{t}: t \geq 0\right\}$ denote the occupation time of the ISDSM defined by (3.14). Then $Z_{t}$ is a.s. absolutely continuous and the corresponding local time is given by

$$
\begin{aligned}
z(b, t)= & \int_{\mathbb{R}} \int_{W_{0}} N_{0}(d a, d w) \int_{0}^{t} w(u) l_{0, a}(b, d u) \\
& +\int_{0}^{t} \int_{\mathbb{R}} \int_{0}^{q\left(Y_{s}, a\right)} \int_{W_{0}} N_{1}(d s, d a, d u, d w) \int_{s}^{t} w(v-s) l_{s, a}(b, d v) .
\end{aligned}
$$

Moreover, the two parameter process $\{z(b, t): b \in \mathbb{R}, t \geq 0\}$ has a version which is Hölder continuous of order $\alpha$ for every $\alpha<1 / 2$.

Proof. Let $\left\{Y_{t}^{(n)}: t \geq 0\right\}$ be defined by the right hand side of (3.13) with $q\left(Y_{s}, a\right)$ replaced by $\eta_{n}(s, a):=n \wedge q\left(Y_{s}, a\right)$. From Theorem 4.1 we know that the occupation time of $\left\{Y_{t}^{(n)}: t \geq 0\right\}$ is absolutely continuous with density given by

$$
\begin{aligned}
z_{n}(b, t)= & \int_{\mathbb{R}} \int_{W_{0}} N_{0}(d a, d w) \int_{0}^{t} w(u) l_{0, a}(b, d u) \\
& +\int_{0}^{t} \int_{\mathbb{R}} \int_{0}^{\eta_{n}(s, a)} \int_{W_{0}} N_{1}(d s, d a, d u, d w) \int_{s}^{t} w(v-s) l_{s, a}(b, d v)
\end{aligned}
$$

which has a Hölder continuous version of order $\alpha$. The same conclusion is clearly true for the occupation time of $\left\{Y_{t}: t \geq 0\right\}$ on the event $\left\{\sup _{0 \leq s \leq t} q\left(Y_{s}, a\right) \leq n\right\}$. By Chebyshev's inequality and the assumption on $q(\cdot, \cdot)$ is easy to show that

$$
\mathbf{P}\left\{\sup _{0 \leq s \leq t} q\left(Y_{s}, a\right) \geq n\right\} \leq \frac{K}{n} \mathbf{E}\left[1+\sup _{0 \leq s \leq t}\left\langle\phi_{p}, Y_{s}\right\rangle^{2}\right]
$$

By Proposition 3.1, the right hand side tends to zero as $n \rightarrow \infty$. Then we have the desired result.

\section{$5 \quad$ Scaling limit theorems}

Scaling limit theorems of SDSM without immigration were investigated in Refs. [5, 9]. A direct generalization of the limit theorem of Ref. [5] to the ISDSM was given in Ref. 6]. 
In this section, we prove two scaling limit theorems for the ISDSM. The limit processes obtained here are different from those in the previous work. As consequences, we also obtain scaling limits for the corresponding local time. For simplicity we assume $Y_{0}=0$ and focus on the influence of the immigration.

Let $\overline{\mathbb{R}}=\mathbb{R} \cup\{\infty\}$ be the one-point compactification of the real line and let $M(\overline{\mathbb{R}})$ be the space of Borel measures on $\overline{\mathbb{R}}$. We fix a metric on $M(\overline{\mathbb{R}})$ compatible with the weak convergence and regard $M(\mathbb{R})$ as a subspace of $M(\overline{\mathbb{R}})$ comprising measures supported by $\mathbb{R}$. Then a metric on $M_{p}(\mathbb{R})$ can be defined through the isomorphism

$$
\Phi_{p}: \mu(d x) \mapsto \phi_{p}(x) \mu(d x)
$$

between $M_{p}(\mathbb{R})$ and $M(\mathbb{R})$. Let $C\left([0, \infty), M_{p}(\overline{\mathbb{R}})\right)$ be the space of continuous paths from $[0, \infty)$ to $M_{p}(\overline{\mathbb{R}})$ endowed with the topology of locally uniform convergence.

We first assume $p>1$ so the Lebesgue measure $\lambda$ belongs to $M_{p}(\mathbb{R})$. Let $q(\cdot, \cdot)$ be a bounded Borel function on $M_{p}(\mathbb{R}) \times \mathbb{R}$ satisfying the local Lipschitz condition (3.3). Suppose that $\{W(d s, d y)\}$ and $\left\{N_{1}(d s, d a, d u, d w)\right\}$ are given as in (2.a) and (2.d) with $m$ replaced by $\lambda$. Let $\left\{x_{r, a}(t): t \geq r\right\}$ be defined by (2.12) and let $\left\{Y_{t}: t \geq 0\right\}$ be the solution of

$$
Y_{t}=\int_{0}^{t} \int_{\mathbb{R}} \int_{0}^{q\left(Y_{s}, a\right)} \int_{W_{0}} w(t-s) \delta_{x_{s, a}(t)} N_{1}(d s, d a, d u, d w) .
$$

For any integer $k \geq 1$ let $Y_{t}^{k}(d x)=k^{-2} Y_{k^{2} t}(k d x)$.

Theorem 5.1 Suppose that $q(\nu, a) \rightarrow q(\infty)$ as $|a| \rightarrow \infty$ for all $\mu \in M_{p}(\mathbb{R})$. Then, as $k \rightarrow$ $\infty,\left\{k^{-1} Y_{t}^{k}: t \geq 0\right\}$ converges to $\{q(\infty) t \lambda: t \geq 0\}$ in probability on $C\left([0, \infty), M_{p}(\mathbb{R})\right)$.

Proof. In this and the following proofs, we write " $=d$ " for the equivalence in distribution of two processes. We shall also consider some new Poisson random measures which might be defined on some extensions of the original probability space.

Step 1. Let $\phi \in C_{p}^{2}(\mathbb{R})$. From (5.2) we have

$$
\left\langle\phi, Y_{t}^{k}\right\rangle=\int_{0}^{t} \int_{\mathbb{R}} \int_{0}^{q\left(Y_{k^{2}}, k b\right)} \int_{W_{0}} w_{k}(t-s) \phi\left(x_{s, b}^{k}(t)\right) N_{1}\left(k^{2} d s, k d b, d u, d w\right),
$$

where $w_{k}(t-s)=k^{-2} w\left(k^{2}(t-s)\right)$ and $\left\{x_{s, b}^{k}(t): t \geq s\right\}$ is the unique solution of

$$
x(t)=b+\int_{s}^{t} \int_{\mathbb{R}} h_{k}(y-x(u)) k^{-3 / 2} W\left(k^{2} d u, k d y\right), \quad t \geq s
$$

with $h_{k}(z)=\sqrt{k} h(k z)$. It is easy to see that $k^{-3 / 2} W\left(k^{2} d u, k d y\right)$ is a white noise based on the Lebesgue measure and $N_{1}\left(k^{2} d s, k d b, d u, d w\right)$ is Poisson random measure with intensity $k^{3} d s d b d u \mathbf{Q}_{\kappa}(d w)$. By the scaling property of the $\sigma$-branching diffusion it is easy to check 
that $\left\{w_{k}(t): t \geq 0\right\}$ under $k^{2} \mathbf{Q}_{\kappa}$ has the same law as $\{w(t): t \geq 0\}$ under $\mathbf{Q}_{\kappa}$. From (5.3) we get

$$
\left\langle\phi, Y_{t}^{k}\right\rangle={ }_{d} \int_{0}^{t} \int_{\mathbb{R}} \int_{0}^{q\left(Y_{k^{2}}, k b\right)} \int_{W_{0}} w(t-s) \phi\left(x_{s, b}^{k}(t)\right) N_{k}(d s, d b, d u, d w),
$$

where $N_{k}(d s, d b, d u, d w)$ is Poisson random measure with intensity $k d s d b d u \mathbf{Q}_{\kappa}(d w)$. Let $\psi_{k}=k^{-1} \phi$. Since $\mathbf{Q}_{\kappa}[w(t)]=1$ for every $t>0$, from (5.4) we have

$$
\begin{aligned}
\mathbf{E}\left[\left\langle\psi_{k}, Y_{t}^{k}\right\rangle\right] & =\mathbf{E}\left[\int_{0}^{t} d s \int_{\mathbb{R}} d b \int_{W_{0}} q\left(Y_{k^{2} s}, k b\right) w(t-s) \phi\left(x_{s, b}^{k}(t)\right) \mathbf{Q}_{\kappa}(d w)\right] \\
& =\int_{0}^{t} d s \int_{\mathbb{R}} \mathbf{E}\left[q\left(Y_{k^{2} s}, k b\right) \phi\left(x_{s, b}^{k}(t)\right)\right] d b \\
& =\int_{0}^{t} d s \int_{\mathbb{R}} d b \int_{\mathbb{R}} \mathbf{E}\left[q\left(Y_{k^{2} s}, k b\right) \mid x_{s, b}^{k}(t)=z\right] \phi(z) g_{\rho(0)(t-s)}(b, z) d z,
\end{aligned}
$$

where $g_{u}(b, z)$ denotes the density of the heat kernel. By dominated convergence we see that

$$
\lim _{k \rightarrow \infty} \mathbf{E}\left[\left\langle\psi_{k}, Y_{t}^{k}\right\rangle\right]=\int_{0}^{t} d s \int_{\mathbb{R}} d b \int_{\mathbb{R}} q(\infty) \phi(z) g_{\rho(0)(t-s)}(b, z) d z=t q(\infty)\langle\phi, \lambda\rangle .
$$

For every $t>0$ we have $\mathbf{Q}_{\kappa}\left[w(t)^{2}\right]=\sigma t$; see e.g. Lemma 3.1 of Ref. [13]. It follows that

$$
\begin{aligned}
& \mathbf{E}\left[\left|\left\langle\psi_{k}, Y_{t}^{k}\right\rangle-\mathbf{E}\left[\left\langle\psi_{k}, Y_{t}^{k}\right\rangle\right]\right|^{2}\right] \\
& =k^{-1} \mathbf{E}\left[\int_{0}^{t} d s \int_{\mathbb{R}} d b \int_{W_{0}} q\left(Y_{k^{2} s}, k b\right) w(t-s)^{2} \phi\left(x_{s, b}^{k}(t)\right)^{2} \mathbf{Q}_{\kappa}(d w)\right] \\
& \leq k^{-1} \sigma\|q\| \int_{0}^{t}(t-s) d s \int_{\mathbb{R}} \mathbf{E}\left[\phi\left(x_{s, b}^{k}(t)\right)^{2}\right] d b \\
& \leq k^{-1} \sigma\|q\| \int_{0}^{t}(t-s) d s \int_{\mathbb{R}} \phi(b)^{2} d b
\end{aligned}
$$

which tends to zero as $k \rightarrow \infty$. That proves $\lim _{k \rightarrow \infty}\left\langle\psi_{k}, Y_{t}^{k}\right\rangle=q(\infty) t\langle\phi, \lambda\rangle$ in probability.

Step 2. From (5.4) and Theorem 2.1 we see that

$$
M_{t}^{k}(\phi):=\left\langle\phi, Y_{t}^{k}\right\rangle-\frac{1}{2} \rho(0) \int_{0}^{t}\left\langle\phi^{\prime \prime}, Y_{s}^{k}\right\rangle d s-k \int_{0}^{t}\left\langle q\left(Y_{k^{2} s}, k \cdot\right) \phi, \lambda\right\rangle d s
$$

is a continuous martingale with quadratic variation process

$$
\left\langle M^{k}(\phi)\right\rangle_{t}=\int_{0}^{t}\left\langle\sigma \phi^{2}, Y_{s}^{k}\right\rangle d s+\int_{0}^{t} d s \int_{\mathbb{R}^{2}} \rho(k(x-y)) \phi^{\prime}(x) \phi^{\prime}(y) Y_{s}^{k}(d x) Y_{s}^{k}(d y) .
$$


Let $\left\{\tau_{k}\right\}$ be a bounded family of stopping times. By (5.5) and (5.6) we have

$$
\begin{aligned}
\mathbf{E}\left[\left|\left\langle\phi, Y_{\tau_{k}+t}^{k}\right\rangle-\left\langle\phi, Y_{\tau_{k}}^{k}\right\rangle\right|^{2}\right] \leq & 3 \mathbf{E}\left[\left|M_{\tau_{k}+t}^{k}(\phi)-M_{\tau_{k}}^{k}(\phi)\right|^{2}\right]+\frac{3}{2} \rho(0) t \int_{0}^{t} \mathbf{E}\left[\left\langle\phi^{\prime \prime}, Y_{\tau_{k}+s}^{k}\right\rangle^{2}\right] d s \\
& +3 k^{2} t \int_{0}^{t} \mathbf{E}\left[\left\langle q\left(Y_{k^{2}\left(\tau_{k}+s\right)}, k \cdot\right) \phi, \lambda\right\rangle^{2}\right] d s
\end{aligned}
$$

where

$$
\mathbf{E}\left[\left|M_{\tau_{k}+t}^{k}(\phi)-M_{\tau_{k}}^{k}(\phi)\right|^{2}\right] \leq \sigma \int_{0}^{t} \mathbf{E}\left[\left\langle\phi^{2}, Y_{\tau_{k}+s}^{k}\right\rangle\right] d s+\|\rho\| \int_{0}^{t} \mathbf{E}\left[\left\langle\phi^{\prime}, Y_{\tau_{k}+s}^{k}\right\rangle^{2}\right] d s
$$

By Proposition 2.1 it is easy to show that

$$
\limsup _{t \rightarrow 0} \mathbf{E}\left[\left|\left\langle\psi_{k}, Y_{\tau_{k}+t}^{k}\right\rangle-\left\langle\psi_{k}, Y_{\tau_{k}}^{k}\right\rangle\right|^{2}\right]=0 .
$$

Another application of Proposition 2.1 shows

$$
\lim _{\alpha \rightarrow \infty} \sup _{k \geq 1} \mathbf{P}\left\{\left\langle\psi_{k}, Y_{t}^{k}\right\rangle \geq \alpha\right\}=0
$$

Then a criterion of Ref. [1] implies that the sequence $\left\{\left\langle\psi_{k}, Y_{t}^{k}\right\rangle: t \geq 0\right\}$ is tight in $C([0, \infty), \mathbb{R})$. Let $\Phi_{p}$ be defined by (5.1). By Theorem 3.7.1 of Ref. [2], $\left\{k^{-1} \Phi_{p} Y_{t}^{k}: t \geq 0\right\}$ is a tight sequence in $C([0, \infty), M(\overline{\mathbb{R}}))$. Now the result of the first step implies that $\left\{k^{-1} \Phi_{p} Y_{t}^{k}: t \geq 0\right\}$ converges to $\left\{t \Phi_{p} \lambda: t \geq 0\right\}$ in probability in $C([0, \infty), M(\overline{\mathbb{R}}))$. Since all the processes live in $C([0, \infty), M(\mathbb{R}))$, the convergence also holds in this smaller space. In other words, $\left\{k^{-1} Y_{t}^{k}: t \geq 0\right\}$ converges to $\{q(\infty) t \lambda: t \geq 0\}$ in probability in $C\left([0, \infty), M_{p}(\mathbb{R})\right)$.

The above theorem implies the following scaling limit theorem for the local time of the ISDSM.

Corollary 5.1 Let $z(\cdot, \cdot)$ denote the local time of $\left\{Y_{t}: t \geq 0\right\}$ given by the second term on the right hand side of (4.11). Under the condition of Theorem [5.1, $z_{k}(t, \cdot):=k^{-5} z\left(k \cdot, k^{2} t\right)$ converges weakly to $q(\infty) t^{2} / 2$ in probability as $k \rightarrow \infty$.

For a finite reference immigration measure, we can prove a limit theorem which gives an interesting random limit process. To describe the limit process we introduce the following concept: A two-parameter process $\left\{y_{r}(t): t \geq r \geq 0\right\}$ is called a restricted coalescing Brownian flow (RCBM flow) with speed $\rho>0$ provided

(5.a) for any fixed $r \geq 0$, the process $\left\{y_{r}(t): t \geq r\right\}$ is a Brownian motion with speed $\rho$ started from $y_{r}(r)=0$;

(5.b) for any fixed $s \geq r \geq 0$, the process $\left\{y_{s}(t)-y_{r}(t): t \geq s\right\}$ is a Brownian motion with speed $2 \rho$ stopped at zero. 
Let $m \in M(\mathbb{R})$ and let $q(\cdot, \cdot)$ be a bounded Borel function on $M(\mathbb{R}) \times \mathbb{R}$ satisfying the local Lipschitz condition (3.3) for $p=0$. In addition, we assume

(5.c) there is a constant $\epsilon>0$ so that $q(\nu, a) \geq \epsilon$ for all $\nu \in M(\mathbb{R})$ and $a \in \mathbb{R}$;

(5.d) $q(\nu, a) \rightarrow q(a)$ as $\langle 1, \nu\rangle \rightarrow \infty$ for all $a \in \mathbb{R}$.

Suppose that $\{W(d s, d y)\}$ and $\left\{N_{1}(d s, d a, d u, d w)\right\}$ are given as in (2.a) and (2.d) and let $\left\{Y_{t}: t \geq 0\right\}$ be defined by (5.2). Let $Y_{t}^{k}(d x)=k^{-2} Y_{k^{2} t}(k d x)$.

Theorem 5.2 Under the above conditions, as $k \rightarrow \infty$, the sequence $\left\{Y_{t}^{k}: t \geq 0\right\}$ converges in distribution on $C([0, \infty), M(\mathbb{R}))$ to

$$
Y_{t}^{\infty}:=\int_{0}^{t} \int_{\mathbb{R}} \int_{0}^{q(a)} \int_{W_{0}} w(t-s) \delta_{x_{s}^{\infty}(t)} N_{1}(d s, d a, d u, d w), \quad t \geq 0,
$$

where $\left\{x_{s}^{\infty}(t): t \geq s\right\}$ is an $R C B M$ flow independent of $\left\{N_{1}(d s, d a, d u, d w)\right\}$ with speed $\rho(0)$.

Proof. Let $\left\{x_{s, b}^{k}(t): t \geq s\right\}$ be defined as in the proof of Theorem 5.1. For any $\phi \in C^{2}(\mathbb{R})$ we have

$$
\left\langle\phi, Y_{t}^{k}\right\rangle=\int_{0}^{t} \int_{\mathbb{R}} \int_{0}^{q\left(Y_{k^{2} s}, a\right)} \int_{W_{0}} w_{k}(t-s) \phi\left(x_{s, a / k}^{k}(t)\right) N_{1}\left(k^{2} d s, d a, d u, d w\right) .
$$

It is easy to see that $N_{1}\left(k^{2} d s, d a, d u, d w\right)$ is a Poisson random measure with intensity $k^{2} d s m(d a) d u \mathbf{Q}_{\kappa}(d w)$. Let $X_{t}^{k}$ be defined by the right hand side of (5.2) with $q\left(Y_{s}, a\right)$ replaced by $q(a)$. Then we have

$$
\left\langle\phi, X_{t}^{k}\right\rangle=\int_{0}^{t} \int_{\mathbb{R}} \int_{0}^{q(a)} \int_{W_{0}} w_{k}(t-s) \phi\left(x_{s, a / k}^{k}(t)\right) N_{1}\left(k^{2} d s, d a, d u, d w\right) .
$$

The scaling property of the $\sigma$-branching diffusion implies that

$$
\left\langle\phi, X_{t}^{k}\right\rangle={ }_{d} \int_{0}^{t} \int_{\mathbb{R}} \int_{0}^{q(a)} \int_{W_{0}} w(t-s) \phi\left(x_{s, a / k}^{k}(t)\right) N_{1}(d s, d a, d u, d w) .
$$

Therefore the distribution of $\left\{\left\langle 1, X_{t}^{k}\right\rangle: t \geq 0\right\}$ on $C\left([0, \infty), \mathbb{R}_{+}\right)$is independent of $k \geq 1$. Indeed, by Theorem 4.1 of Ref. [11] it is easy to see that $\left\{\left\langle 1, X_{t}^{k}\right\rangle: t \geq 0\right\}$ is distributed as the $\sigma$-branching diffusion with immigration that solves the stochastic equation

$$
d z_{t}=\sqrt{\sigma z_{t}} d B_{t}+\langle q, m\rangle d t, \quad z_{0}=0 .
$$

Given $\left(s_{1}, \cdots, s_{m} ; a_{1}, \cdots, a_{m}\right)$, it can be proved as Theorem 2.2 of Ref. [5] that $\left\{\left(x_{s_{1}, a_{1} / k}^{k}(\cdot)\right.\right.$, $\left.\left.\cdots, x_{s_{m}, a_{m} / k}^{k}(\cdot)\right)\right\}$ converges in distribution to $\left\{\left(x_{s_{1}}^{\infty}(\cdot), \cdots, x_{s_{m}}^{\infty}(\cdot)\right)\right\}$. By dominated convergence it is easy to show that $\left\{\left\langle\phi, X_{t}^{k}\right\rangle: t \geq 0\right\}$ converges in finite dimensional distributions to

$$
\left\langle\phi, Y_{t}^{\infty}\right\rangle=\int_{0}^{t} \int_{\mathbb{R}} \int_{0}^{q(a)} \int_{W_{k}} w(t-s) \phi\left(x_{s}^{\infty}(t)\right) N_{1}(d s, d a, d u, d w), \quad t \geq 0 .
$$


On the other hand, from (5.8) and (5.9) it is easy to see that

$$
\left|\left\langle\phi, X_{t}^{k}\right\rangle-\left\langle\phi, Y_{t}^{k}\right\rangle\right| \leq \int_{0}^{t} \int_{\mathbb{R}} \int_{q(a) \wedge q\left(Y_{k^{2} s}, a\right)}^{q(a) \vee q\left(Y_{k^{2}}, a\right)} \int_{W_{0}} w_{k}(t-s)\|\phi\| N_{1}\left(k^{2} d s, d a, d u, d w\right)
$$

and so

$$
\mathbf{E}\left[\left|\left\langle\phi, X_{t}^{k}\right\rangle-\left\langle\phi, Y_{t}^{k}\right\rangle\right|\right] \leq\|\phi\| \int_{0}^{t} d s \int_{\mathbb{R}} \mathbf{E}\left[\left|q(a)-q\left(Y_{k^{2} s}, a\right)\right|\right] m(d a),
$$

By Condition (5.c) we find that $\left\langle 1, Y_{k^{2} s}\right\rangle=k^{2}\left\langle 1, Y_{s}^{k}\right\rangle \rightarrow \infty$ in probability for every $s>0$. Then (5.10) and Condition (5.d) implies that $\left|\left\langle\phi, X_{t}^{k}\right\rangle-\left\langle\phi, Y_{t}^{k}\right\rangle\right| \rightarrow 0$ in probability. Therefore $\left\{\left\langle\phi, Y_{t}^{k}\right\rangle: t \geq 0\right\}$ also converges to $\left\{\left\langle\phi, Y_{t}^{\infty}\right\rangle: t \geq 0\right\}$ in finite dimensional distributions. The tightness of $\left\{Y_{t}^{k}: t \geq 0\right\}$ can be established as in the proof of Theorem 5.1, so the sequence converges to $\left\{Y_{t}^{\infty}: t \geq 0\right\}$ in distribution on $C([0, \infty), M(\mathbb{R}))$.

Corollary 5.2 Let $z(\cdot, \cdot)$ denote the local time of $\left\{Y_{t}: t \geq 0\right\}$. Under the condition of Theorem [5.2, $k^{-4} z\left(k \cdot, k^{2} t\right)$ converges weakly in distribution to

$$
z^{\infty}(\cdot, t):=\int_{0}^{t} \int_{\mathbb{R}} \int_{0}^{q(a)} \int_{W_{0}} N_{1}(d s, d a, d u, d w) \int_{s}^{t} w(v-s) l_{s}^{\infty}(\cdot, d v),
$$

where $\left\{l_{s}^{\infty}(b, u): u \geq s\right\}$ denotes the local time of $\left\{x_{s}^{\infty}(u): u \geq s\right\}$ at $b \in \mathbb{R}$. Moreover, the process $\left\{z^{\infty}(b, t): b \in \mathbb{R}, t \geq 0\right\}$ has a version which is Hölder continuous of order $\alpha$ for every $\alpha<1 / 2$.

Proof. The first part is an immediate consequence of Theorem 5.2. The second part follows by arguments similar to those given in the proof of Theorem 4.1.

\section{Acknowledgements}

Z.L. was supported by NSFC (10121101 and 10525103) and NCET (04-0150). J.X. was supported partially by NSA.

\section{References}

[1] Aldous, D. (1978): Stopping times and tightness. Ann. Probab. 6, 335-340.

[2] Dawson, D.A. (1993): Measure-valued Markov processes. In: Lect. Notes. Math. 1541, 1-260. Springer-Verlag, Berlin. 
[3] Dawson, D.A. and Li, Z.H. (2003): Construction of immigration superprocesses with dependent spatial motion from one-dimensional excursions. Probab. Theory Related Fields 127, 37-61.

[4] Dawson, D.A.; Li, Z.H. and Wang, H. (2001): Superprocesses with dependent spatial motion and general branching densities. Elect. J. Probab. 6, Paper No. 25, 1-33.

[5] Dawson, D.A.; Li, Z.H. and Zhou, X.W. (2004): Superprocesses with coalescing Brownian spatial motion as large scale limits. J. Theoret. Probab. 17, 673-692.

[6] Dôku, I. (2006): A limit theorem of superprocesses with non-vanishing deterministic immigration. Scientiae Mathematicae Japonicae 64, 563-579.

[7] Fu, Z.F. and Li, Z.H. (2004): Measure-valued diffusions and stochastic equations with Poisson process. Osaka J. Math. 41, 727-744.

[8] Li, Z.H.; Lu, G.H. and Wang, H. (2004): Immigration superprocesses with dependent spatial motion and non-critical branching. Chinese J. Contemp. Math. 25, 405-416.

[9] Li, Z.H., Wang, H. and Xiong, J. (2004): A degenerate stochastic partial differential equation for superprocesses with singular interaction. Probab. Theory Related Fields 130, 1-17.

[10] Li, Z.H.; Wang, H. and Xiong, J. (2005): Conditional log-Laplace functionals of immigration superprocesses with dependent spatial motion. Acta Appl. Math. 88, 143-175.

[11] Pitman, J. and Yor, M. (1982): A decomposition of Bessel bridges. Z. Wahrsch. verw. Geb. 59, 425-457.

[12] Revuz, D. and Yor, M. (1991): Continuous Martingales and Brownian Motion. SpringerVerlag, Berlin.

[13] Shiga, T. (1990): A stochastic equation based on a Poisson system for a class of measurevalued diffusion processes. J. Math. Kyoto Univ. 30, 245-279.

[14] Walsh, J.B. (1986): An Introduction to Stochastic Partial Differential Equations. In: Lect. Notes Math. 1180, 265-439. Springer-Verlag, Berlin.

[15] Wang, H. (1997): State classification for a class of measure-valued branching diffusions in a Brownian medium. Probab. Theory Related Fields 109, 39-55.

[16] Wang, H. (1998): A class of measure-valued branching diffusions in a random medium. Stochastic Anal. Appl. 16, 753-786. 